\title{
Controlling the sensitivity of a Non-adiabatic tapered optical fiber for measuring the refractive index using all fiber Sagnac loop Interferometer
}

\author{
M. I. Zibaiia ${ }^{a, b, ~ *, ~ P . ~ A . ~ S . ~ J o r g e ~}{ }^{a}$, O. Frazão a and H. Latifi ${ }^{b}$ \\ ${ }^{a}$ Instituto de Engenharia de Sistemas e Computadores do Porto (INESC Porto), Rua do Campo \\ Alegre, 687. 4169-007 Porto, Portugal; \\ ${ }^{\mathrm{b}}$ Laser and Plasma Research Institute, Shahid Beheshti University, 1983963113, Tehran, Iran.
}

\begin{abstract}
A single-mode non-adiabatic tapered optical fiber (NATOF) sensor was inserted into a Sagnac loop interferometer allowing tuning its sensitivity to refractive index (RI) by use of polarization control. By adjusting any polarization controllers inserted in the Sagnac loop interferometer, various cladding modes are selectively excited in each arm of the interferometric taper resulting into different phase changes for the clockwise and counterclockwise beams. By this method, sensitivity of the sensor for RI in the range from 1.3380 to 1.3510 was tuned between $876.24 \mathrm{RIU} / \mathrm{nm}$ to 1233.07 RIU/nm.
\end{abstract}

Keywords: Non-adiabatic tapered, optical fiber sensor, refractive index, Sagnac loop interferometer

\section{INTRODUCTION}

In many areas of biophysics, biochemistry and biomedicine measurements are based on two fundamental concepts, namely spectroscopy (fluorescence and absorption) and evanescent field interaction [1-3]. The second concept is based on the partial overlap of the evanescent guided electromagnetic wave with a medium whose refractive index (RI) is measured. The measurement of changes in RI has applications such as the study of a molecular structure and the identification of organic compounds in the medical, pharmaceutical, plastic, food, industrial fluid, petrochemical and beverage industries [4].

Tapered fiber-based sensors have recently been reported as simple and cost-effective alternatives among many types of fiber-optic sensors [5-13]. Some of the tapered fiber-based sensors reported include biconical fiber tapers that can measure stress, temperature, and RI [5-6]; make a Mach-Zehnder interferometer [7], a Michelson interferometer [8], a tapered long period grating [9]; a tapered sensor based on absorption [10]; a tapered fiber humidity sensor [11]; and a temperature-independent strain sensor made from a tapered holey optical fiber [12,13].

Optical fiber Sagnac loop interferometers (FSLIs) have been developed for gyroscopes and other sensor applications due to their unique advantages, such as simple design, ease of manufacture, lower susceptibility to environmental pickup noise in comparison to other types of fiber optic sensors, high sensitivity to external perturbation change, polarization independence to input light, low insertion loss, electromagnetic immunity, and high measuring precision [14]. Usually, the FSLI is based on a high-birefringent fiber to obtain an optical path difference between the fast and the slow axes. Recently, intermodal interference in a small core microstructure fiber loop mirror was demonstrated [15].

In this work a new configuration based on a NATOF inserted in a FSLI used as an optical refractometer is demonstrated. It is demonstrated the possibility of tuning the device sensitivity to RI variations of the surrounding medium by using polarization control. In this case, the interference is guaranteed by excitation of different high order modes.

\footnotetext{
*m_zibaye@ sbu.ac.ir
}

International Conference on Applications of Optics and Photonics, edited by Manuel F. M. Costa

Proc. of SPIE Vol. 8001, 800144 - (C) 2011 SPIE · CCC code: 0277-786X/11/\$18 · doi: 10.1117/12.892102 


\section{PRINCIPLE OF SENSOR}

When the optical fiber is tapered, the core-cladding interface is redefined in such a way that the light propagation inside the core penetrates into the cladding, which plays the role of the new core, and the external medium is the new cladding [16]. The tapered fiber consists of a conical segment where the diameter of the fiber gradually decreases, a relatively long taper waist section where the diameter of the fiber is small and uniform, and a second conical segment with its waist merging into the single mode fiber again. Depending on the pulling conditions (pulling speed, length of the heated zone, pulling temperature, etc) one can fabricate biconical tapers with different shapes and properties. Fiber tapers may be divided into two distinct categories: adiabatic and nonadiabatic. A tapered fiber can be considered adiabatic if the main portion of the power remains in the fundamental mode $\left(\mathrm{LP}_{01}\right)$ and does not couple to higher order modes as it propagates along the taper. To avoid the coupling between the fundamental mode and higher order modes, the taper local length scale must be much larger than the coupling length between these two modes. In other words, the relative local change in the taper radius has to be very small. It has been shown that non-adiabatic fiber tapers can be made so that coupling occurs primarily between the fundamental mode of the unpulled fiber and the first two modes of the taper waveguide $\left(\mathrm{LP}_{01}, \mathrm{LP}_{02}\right)$, where due to the large difference of the RIs of air and glass, the taper normally supports more than one mode $[4,16]$. The light propagates at the air-cladding interface of the tapers waist region in which case the SMF was converted to the MMF. The result of back and forth coupling between the single mode of the fiber and the two (or more) modes of the taper is oscillations in the spectral response of the taper. Which means the transmission is high for certain wavelengths and low for others (typically, transmission versus wavelength shows periodic behavior) [4-5]. Therefore, with the NATOF, one achieves a modal Mach-Zehnder interferometer. The efficiency of this last coupling is dependent on the relative phase of the participating modes. When there are only two modes, the relative phase is $\Delta \varphi=\Delta \beta L$, where $\Delta \beta$ and $L$ are the difference in propagation constants of the $\mathrm{LP}_{01}$ and $\mathrm{LP}_{02}$ cladding modes accumulated in the beating region and the interaction length along the taper, respectively. Therefore, the spectral response of the taper will shift correspondingly by changing the above terms. For instance, if the RI of the surrounding environment of the taper changes, the difference in propagation constants and the relative phase would be modified leading to a shift of the spectral response. It is thus obvious that one can build sensitive RI sensors based on the NATOF [4-5]. This interferometric device is appealing for environmental sensing also because to a certain extent it is possible to tune its sensitivity to external RI variations by selecting the order of the cladding mode.

\section{SENSOR FABRICATION AND EXPERIMENTAL SETUP}

\subsection{Nonadiabatic taper fabrication}

Figure 1 illustrates the heat pulling system utilizing a $\mathrm{CO}_{2}$ laser to fabricate the NATOF. The $\mathrm{CO}_{2}$ laser beam is focused via a $\mathrm{Zn}-\mathrm{Se}$ lens of $2.5 \mathrm{~cm}$ focal length. The resultant spot size is about $120 \mu \mathrm{m}$ on the fiber length. After incidence in

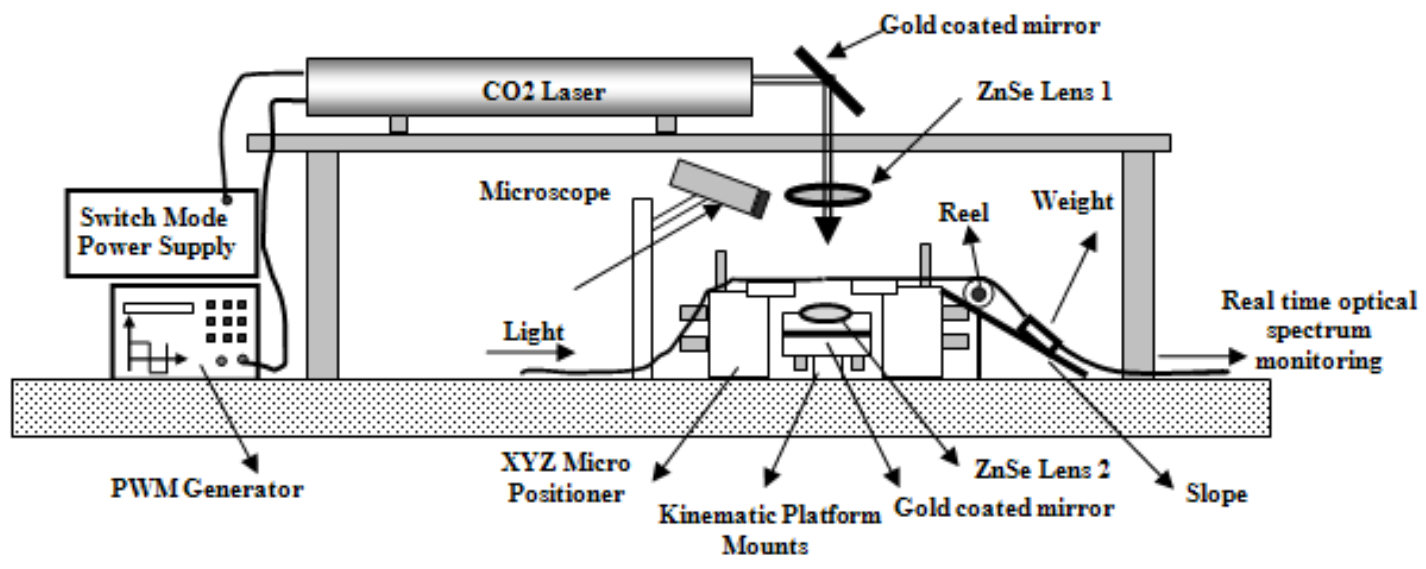

Figure 1. Schematic of the fabrication system for a tapered fiber by the heat-pulling method. 


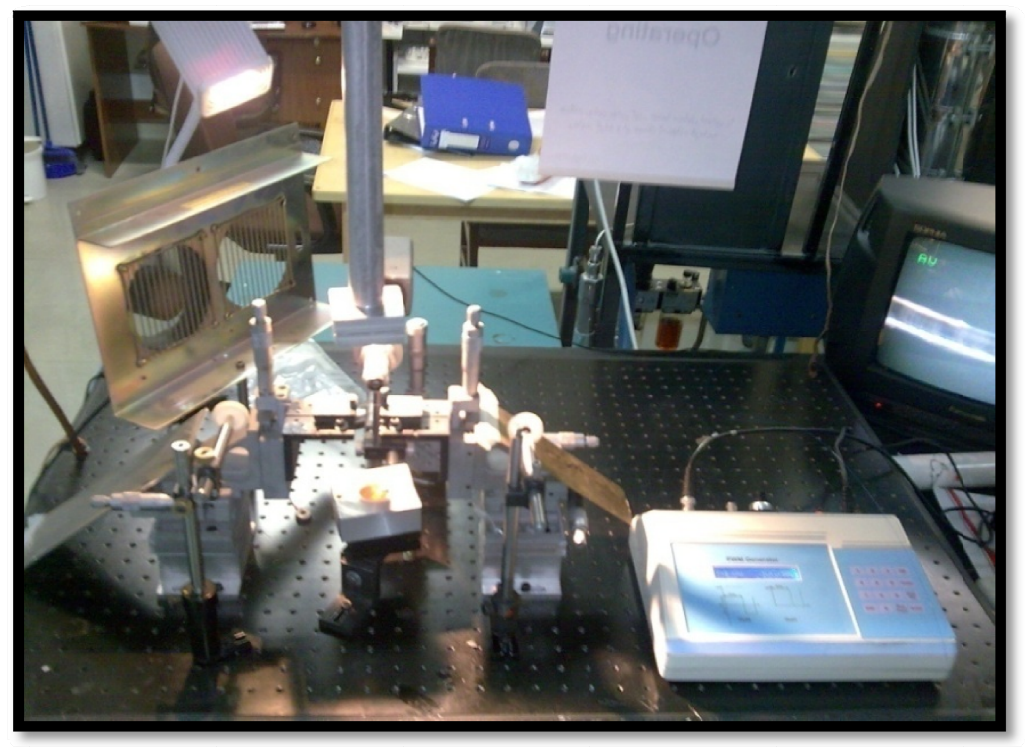

Figure 2. Image of the fabrication system for a tapered fiber by $\mathrm{CO}_{2}$ laser.

the SMF, the laser beam propagates through the $\mathrm{Zn}-\mathrm{Se}$ lens 2 and is focused at a gold-coated mirror; the reflected beam is then focused on the SMF. Therefore by using this setup, both sides of the SMF are heated. One end of the fiber was fixed on a translation stage and the other end was attached to a $5.12 \mathrm{~g}$ weight which is on a slope so that the fiber was subjected to a constant tension during the laser irradiation. The value of the utilized weight has been optimized to fabricate tapers with desired structures. The geometric characteristic of the fabricated taper depends on the power, number of pulses, beam shape and duty cycle of the $\mathrm{CO}_{2}$ laser. A microscope was employed to monitor the tapering process. The image of setup is shown in figure 2. The present experiment was performed using a NATOF with a taper length between 17 and $20 \mathrm{~mm}$ and an average taper waist diameter in the range of 6-8 $\mu \mathrm{m}$.

To measure the transmission characteristics of the fabricated tapered fiber, light from a wide bandwidth source was launched into the taper via a short SMF pigtail. The output spectrum from the tapered fiber was observed by an optical spectrum analyzer (OSA).

\subsection{Experimental setup}

Figure 3 illustrates the experimental setup which consists of an optical broadband source, a fiber loop mirror, a NATOF and an optical spectrum analyzer (OSA) with a maximum resolution of $10 \mathrm{pm}$. The optical source is an erbium doped broadband source, with a central wavelength of $1550 \mathrm{~nm}$ and a spectral bandwidth of $100 \mathrm{~nm}$. The taper- fiber Sagnac loop interferometers (TFSLI) is formed by a $3 \mathrm{~dB}$ optical coupler with low insertion loss, two polarization controllers (PCs) and a NATOF which is placed between the PCs. Light travels towards the $3 \mathrm{~dB}$ coupler at port 1, and is

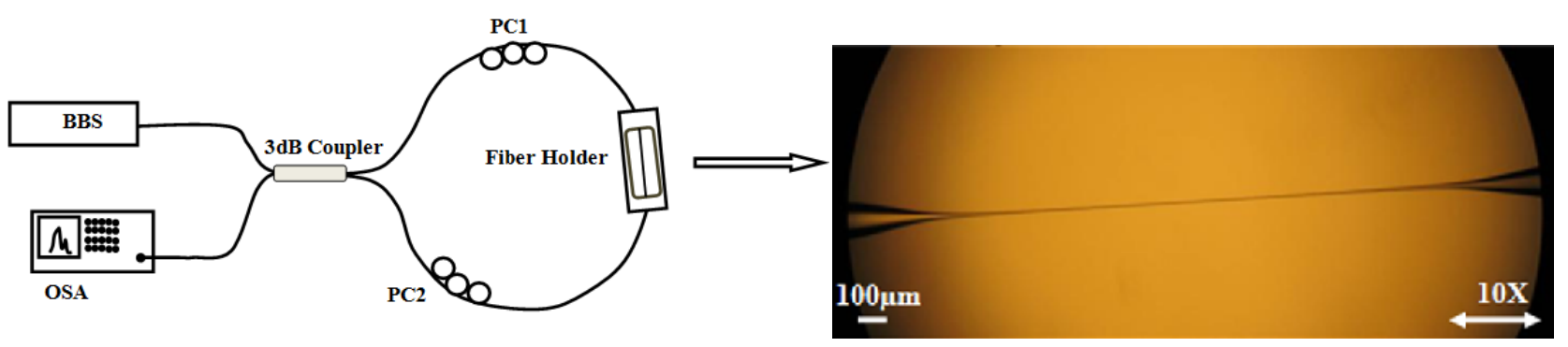

Figure 3. Schematic of a NATOF in fiber loop mirror and a image of non-adiabatic tapered fiber with a taper length of $843 \mu \mathrm{m}$, and waist diameter of $11 \mu \mathrm{m}$. 
split into two counter- propagating beams having fifty percent of the input power and a relative phase difference of $\pi / 2$. Because the coupling efficiency to the different modes supported by the NATOF is polarization dependent, using this configuration it becomes possible to optimize the visibility of the fringe pattern and even its periodicity. This can be done simply by adjusting the PCs and therefore changing the way high order cladding modes are excited. Using two PCs allow us to create different coupling conditions for each of the counter propagating beams breaking the symmetry of the fiber loop resulting in an interferometric output that is very sensitive to external RI.

\section{RESULTS AND DESCUSION}

To measure the sensitivity of our tapered fibers to the RI of the surrounding environment, the fiber holder was filled with different concentrations of salt solution and its optical transmission was measured. The RI of the solutions tested was in the range from 1.3380 to 1.3510 . After each measurement, a micropipette was used to remove the solution from the fiber holder, and the device was washed three times with distilled water.

In the experimental setup, the NATOF was fixed under tension in the fiber holder preventing its bending inside the fiber holder. As a result strain was a constant parameter during the experiment. During the tests the temperature of the solution

was kept approximately constant showing changes smaller than $\pm 0.5^{\circ} \mathrm{C}$. These fluctuations lead to wavelength shifts of about $\pm 0.01 \mathrm{~nm}$ which is the OSA maximum accuracy. Therefore, during the test, the NATOF showed no cross sensitivity to temperature and strain.

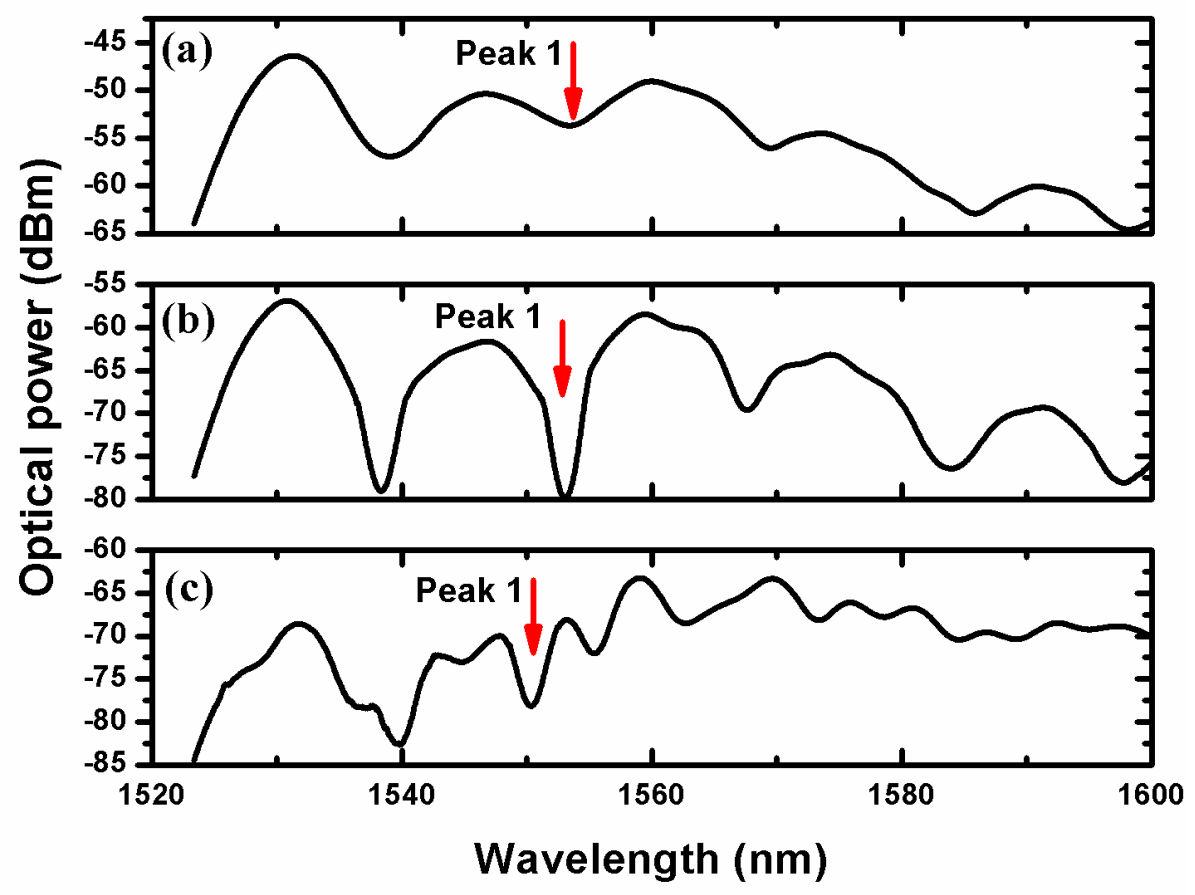

Figure 4. Spectral response of the NATOF in water solution at room temperature for three different positions (a), (b), and (c) of the polarization controllers.

By varying any of the PCs, the coupling conditions to different cladding modes are changed in each arm of the TFSLI resulting in different optical paths for clockwise and counterclockwise beams. In figure 4 the results obtained for three different positions of the PCs are presented. Comparison of figure $4 \mathrm{a}$ ) and figure $4 \mathrm{~b}$ ) shows that the amount of power coupled to each mode can be controlled enabling visibility enhancement. In addition, figure 4c) shows a fringe pattern with a smaller period, demonstrating the possibility to excite different higher order modes. In this case a lower visibility is obtained due to low mode power.

We have experimentally measured the wavelength shift of transmission peak 1 (as depicted in Fig. 4) at different RI of the surrounding environment for different positions of the PCs. 


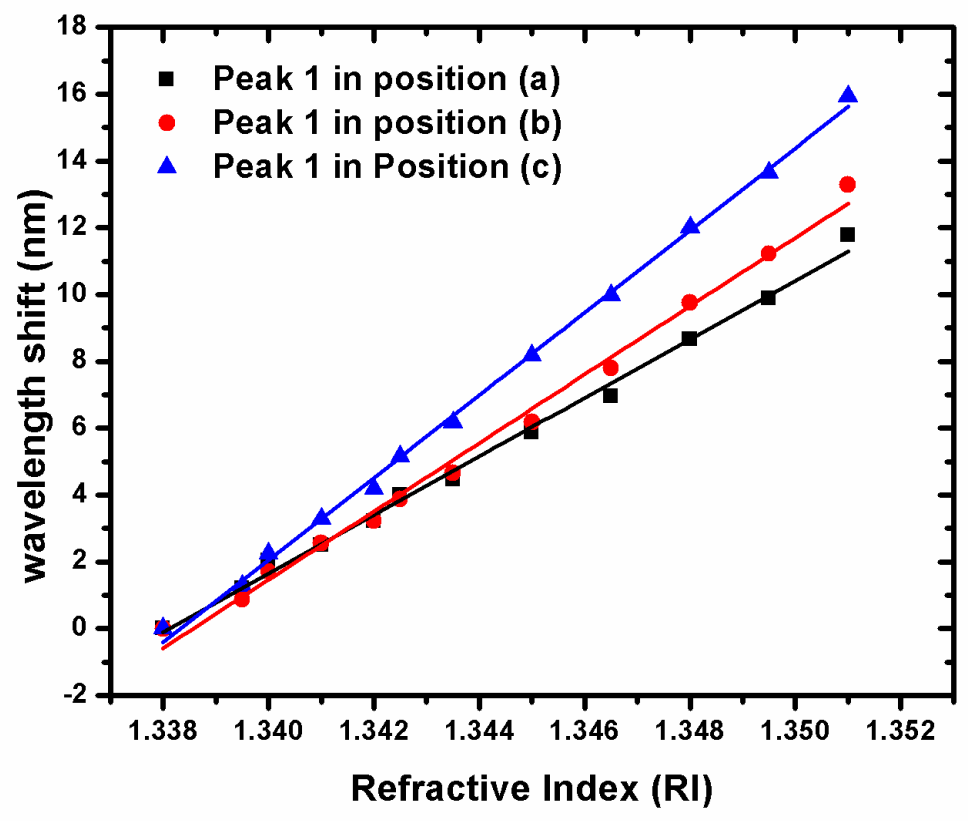

Figure 5. Wavelength shift of transmission peak 1 versus the refractive index for three different positions of the polarization controllers in (a), (b), and (c).

Figure 5 shows the plots of the wavelength shift of transmission peak 1 versus the RI for the three different positions of the PCs in (a), (b), and (c). It is apparent that, in all cases, peak 1 is linearly shifted towards longer wavelength as the RI increases.

The calibration curves obtained allowed to estimate correlation coefficients, $\mathrm{R}^{2}$, of $0.995,0.993$ and 0.998 and sensitivities of $876.24 \mathrm{~nm} / \mathrm{RIU}, 1024.0 \mathrm{~nm} / \mathrm{RIU}$, and $1233.07 \mathrm{~nm} / \mathrm{RIU}$ for positions (a), (b), and (c) of the PCs, respectively. Comparing the sensitivities obtained in cases (b) and (c) with case (a) an improvement of $17 \%$ and $41 \%$ can be estimated, respectively. This sensitivity enhancement agrees with the fact that high order cladding modes are typically more sensitive to RI.

The configuration presented here offers simplicity of design and an enhancement of almost an order of magnitude in sensitivity when compared to previous work of a Hi-Bi FLM using chemical etched $D$-type fiber for RI measurement [14]. In addition, in this particular case, the sensitivity of the TFLM can be controlled and showing great potential in several applications, particularly to monitor chemical and biological parameters in natural environments.

\section{SUMMARY AND CONCLUSION}

In conclusion, a single-mode nonadiabatic tapered optical fiber (NATOF) sensor was inserted into a optical fiber Sagnac loop interferometers (FSLIs) enabling the control of its sensitivity to refractive index (RI). In particular, by adjustment of polarization, sensitivity of the sensor for RI in the range from 1.3380 to 1.3510 could be enhanced by as much as $41 \%$ to $1233.07 \mathrm{~nm} / \mathrm{RIU}$. It should be emphasized that these figures are not any limiting values as sensitivity and detection limit of NATOF sensor can be enhanced by increasing of the taper length and decreasing of the waist diameter. In this context, the configuration presented here is a simple and cost-effective way to further enhance the NATOF sensitivity and versatility, having great potential in label free optical biosensor applications such as medical diagnostics, drug screening, food safety and environment monitoring. 


\section{REFERENCES}

[1] Kim, D., W., Zhang, Y., Cooper, K., L., and Wang, A., "Fibre-optic interferometric immuno-sensor using long period grating," Electron.Lett. 42( 6), 324-325 (2006).

[2] Caldas, P., Jorge, P., A., S., Araújo, F., M., Ferreira, L., A., Marques, M., B., Rego, G., and Santos, J., L., "Fiber modal Michelson interferometers with coherence addressing and heterodyne interrogation," Optical Eng. 47(4) (2008).

[3] Tang, J., L., Cheng, S., F., Hsu, W., T., Chiang, T., Y., and Chau, L., K., "Fiber-optic biochemical sensing with a colloidal gold-modified long period fiber grating," Sens. Actuators B, 119 (1), 105-109 (2006).

[4] Zibaii, M., I., Latifi, H., Karami, K., Gholami, M., Hosseini, S., M., and Ghezelayagh, M., H., "Non-adiabatic tapered optical fiber sensor for measuring the interaction between $\alpha$-amino acids in aqueous carbohydrate solution," J. Meas. Sci. Technol. 21(10), (2010).

[5] Kieu, K., Q., and Mansuripur, M., “Biconical fiber taper sensor,” J. IEEE Photonics Techno. Lett.. 18( 21), 2239-41 (2006).

[6] Datta, P., Matias, C., Aramburu, C. , Bakas, A., Lopez-Amo, M., and Oton, J., M., "Tapered optical fiber temperature sensor," Microwave Opt. Technol. Lett. 11(2), 93-95 (1996).

[7] Lu, P., Men, L., Sooley, K., and Chen, Q., "Tapered fiber Mach- Zehnder interferometer for simultaneous measurement of refractive index and temperature," Appl. Phys. Lett. 94(13), (2009).

[8] Tian, Z., Yam, S., S., H., and Loock, H., P., "Refractive index sensor based on an abrupt taper Michelson interferometer in a single-mode fiber," Opt. Lett., 33(10), 1105-1107 (2008).

[9] Pilla, P., Giordano, M., Korwin-Pawlowski, M., L., Bock, W., J., "sensitivity characteristics tunig in the tapered long period grating by nanocoating," J. IEEE Photonics, 19( 19), 1517-19 (2007).

[10] Leung, A., Shankar, P., M., and Mutharasan, R., "Real-time monitoring of bovine serum albumin at femtogram $/ \mathrm{mL}$ levels on antibodyimmobilized tapered fibers," Sens. Actuators B, 123( 2), 888-895 (2007).

[11] Tazawa, H.,. Kanie, T., and Katayama, M., "Fiber-optic coupler based refractive index sensor and its application," Appl.Phys. Lett. 91( 11), 113901 (2007).

[12] Corres, J., M., Arregui, F., J., and Matias, I., R., "Design of humidity sensor based on tapered optical fibers," $J$. Lightwave Technol., 24( 11), 4329-4336 (2006).

[13] Villatoro, J., Minkovich, V., P., and Monzn-Hernndez, D., “ Temperature-independent strain sensor made from tapered holy optical fiber," Opt. Lett., 31( 3), 305-307 (2006).

[14]Frazão, O., Baptista, J., M., and Santos, J., L., "Recent advances in high-birefringence fiber loop mirror sensors," $J$. Sensors, 7, 2970-83 (2007).

[15] André, R., M., Marques, M., B., Roy, P., Frazão, O., "Fiber loop mirror using a small core microstructured fiber for strain and temperature discrimination", J. IEEE Photonics Techno. Lett., 22(15), 1120-1122 (2010).

[16] Snyder, A., W., Love, J., [Optical Waveguide Theory]. London, U.K.: Chapman \& Hall (1983). 